\title{
Medical history of coronary artery disease and time to electrocardiogram in the emergency department: a real-life, single-center, retrospective analysis
}

Lukas Andreas Heger ${ }^{1 *}$, , Tina Glück ${ }^{1}$, Klaus Kaier ${ }^{2,3}$, Marcus Hortmann ${ }^{1}$, Marina Rieder ${ }^{1}$, Patrick M. Siegel ${ }^{1}$, Philipp Diehl ${ }^{1}$, Tobias Wengenmayer ${ }^{1}$, Christoph B. Olivier ${ }^{1}$, Christoph Bode ${ }^{1}$, Hans-Joerg Busch ${ }^{4}$,

Daniel Duerschmied ${ }^{1}$ and Ingo Ahrens ${ }^{5}$

\begin{abstract}
Background: Timely acquisition of 12-lead Electrocardiogram (ECG) in the emergency department (ED) is crucial and recommended by current guidelines.

Objectives: To evaluate the association of medical history of coronary artery disease (hCAD) on door-to-ECG time in the ED.

Methods: In this single center, retrospective cohort study, patients admitted to ED for cardiac evaluation were grouped according to hCAD and no hCAD. The primary outcome was door-to-ECG time. A multivariate analysis adjusted for the cofounders sex, age, type of referral and shift was performed to evaluate the association of hCAD with door-to-ECG time.
\end{abstract}

Results: 1101 patients were included in this analysis. 362 patients (33\%) had hCAD. Patients with hCAD had shorter door-to-ECG time (20 min. [Inter Quartile Range [IQR] 13-30] vs. 22 min. [IQR 14-37]; $p<0.001$ ) when compared to patients with no hCAD. In a multivariable regression analysis hCAD was significantly associated with a shorter door-toECG time ( -3 min $[p=0.007 ; 95 \%$ confidence Interval $[C l]-5.16$ to $-0.84 \mathrm{~min}]$ ).

Conclusion: In this single center registry, hCAD was associated with shorter door-to-ECG time. In patients presenting in ED for cardiac evaluation, timely ECG diagnostic should be facilitated irrespective of hCAD.

Keywords: Cardiac Evaluation, Coronary artery disease, Chest pain unit, Door-to-ECG time, Door-to-coronaryangiography, Emergency department

*Correspondence: lukas.heger@uniklinik-freiburg.de

${ }^{1}$ Department of Cardiology and Angiology I, Heart Center Freiburg

University, Faculty of Medicine, University of Freiburg, Freiburg, Germany

Full list of author information is available at the end of the article

\section{Background}

Chest pain is one of the most common causes for referral to emergency departments (ED) worldwide and is challenging through its heterogeneous causes [1,2]. Simultaneously, limited access to primary care and patient perceived urgency result in a trend towards increased annual ED attendance [3]. This amounts to an increasing number of patients in need of rapid evaluation to determine whether any life-threatening disease for example

(c) The Author(s) 2021. Open Access This article is licensed under a Creative Commons Attribution 4.0 International License, which permits use, sharing, adaptation, distribution and reproduction in any medium or format, as long as you give appropriate credit to the original author(s) and the source, provide a link to the Creative Commons licence, and indicate if changes were made. The images or other third party material in this article are included in the article's Creative Commons licence, unless indicated otherwise in a credit line to the material. If material is not included in the article's Creative Commons licence and your intended use is not permitted by statutory regulation or exceeds the permitted use, you will need to obtain permission directly from the copyright holder. To view a copy of this licence, visit http://creativecommons.org/licenses/by/4.0/. The Creative Commons Public Domain Dedication waiver (http://creativeco mmons.org/publicdomain/zero/1.0/) applies to the data made available in this article, unless otherwise stated in a credit line to the data. 
one of the "big five" of acute chest pain (aortic dissection, pericarditis with tamponade, esophageal perforation, pulmonary embolus and tension pneumothorax) may be present [4].

In patients with acute myocardial infarction (AMI; "acute myocardial infarction") a prompt recognition is vital, since the beneficial effects of therapy are greatest when performed soon after symptom presentation [5-7]. Recent clinical evidence emphasize that especially highrisk patients profit from rapid diagnostic and therapy $[8$, 9]. Therefore, beside clinical history and cardiac markers, early acquisition of a 12-lead electrocardiogram (ECG) in the ED gains importance especially when it comes to the decision for reperfusion therapy. Consequently, a 10 min target for door-to-ECG time is recommended in the majority of national guidelines $[7,8]$. Nonetheless, several studies have shown that only one-third of patients with Acute Coronary Syndrome (ACS) receive ECG acquisition attained the target of 10 min after admission. Although societies have made suggestions for performing ECG in the ED, only a minority of the literature addresses how to adhere to the $10 \mathrm{~min}$ goal. In the study at hand, we strive to single out clinical factors associated with door-to-ECG time.

Patients with diagnosed coronary artery disease (CAD) have an about $5-10 \%$ risk for recurrent cardiovascular events each year and recent guidelines label them to be high-risk patients $[10,11]$.

Medical history of CAD is part of several risk scores for risk stratification of patients with chest pain and is among the first clinical data the physician would be confronted with after admission. Therefore we suspected hCAD to influence clinical management [12-15].

This single-center, retrospective cohort study aimed to evaluate secular trends in ED workflow, comparing patients with hCAD and patients with no hCAD admitted for chest pain evaluation for door-to-ECG time and time from beginning of symptoms to admission and time to coronary angiography (CAG).

\section{Methods}

\section{Cohort}

In this single center, retrospective cohort study we screened patients admitted to the ED for cardiac evaluation between April and December 2013 for door-toECG-time and in a subgroup analysis door-to-CAG-time. Patients were grouped for hCAD and no hCAD accordingly. We also compared both groups in a subgroup analysis of patients who received CAG. The protocol of this study conforms to the ethical guidelines of the 1975 Declaration of Helsinki and was henceforth approved by the institutional ethical committee of University of Freiburg (permit numbers EK99/17).

\section{Outcomes}

Primary outcome was the door-to-ECG-time in all included patients. Secondary outcome was the time from initial symptoms to admission and in patients receiving CAG the door-to-CAG-time.

\section{Screening}

Full-text keyword-search of the anonymized ED Database segments: key symptoms at admission, anamnesis and diagnosis; was used to single out patients admitted to ED for cardiac evaluation. Keywords included: chest pain; dyspnoea; angina pectoris (AP); retrosternal chest pain; shortness of breath; ST-elevation myocardial infarction (STEMI); non-ST-elevation myocardial infarction (NSTEMI); acute myocardial infarction (AMI); Acute coronary syndrome; myocardial infarction and heart failure.

\section{Patient characteristics and time points}

All patient clinical characteristics as well as laboratory data were obtained retrospectively from the hospital's electronic database. Baseline Characteristics include age, sex and cardiovascular risk factors such as preexisting diagnoses of diabetes mellitus, hypertension, smoking and family with history of cardiovascular disease.

The time points: "start of symptoms", admission to ED", "ECG at ED" and "Coronary angiography" were assessed using electronic records or documentation in the hospital database.

\section{Admission process}

Patients admitted to ED are referred to a triage area upon arrival for identifying high and low-urgency patients. Patients admitted via emergency doctor skip this process and are immediately transferred to the ward. Assessment by the hospital triage nurse includes sex, age, chief complaint and epitome of the patient history.

If the emergency doctor suspects a transmural myocardial infarction, the patient is referred directly to the catheter lab for acute coronary intervention bypassing the ED.

\section{Statistical consideration}

Continuous patient data were compared using a T-test if found to follow a Gaussian distribution otherwise data underwent a Mann-Whitney U-test. Categorical differences between patient groups were compared using Fishers exact test. Continuous variables are presented as median \pm lower and upper quartiles if found to follow a non-Gaussian distribution and as 
mean \pm standard deviation if found to follow a Gaussian distribution according to the D'Agostino-Pearson omnibus normality test. Categorical patient characteristics are presented as percentages. A multivariable median regression model was established to assess influence of a hCAD on time to ECG. As potential confounders, we took into consideration a predefined number of factors that would be obvious to the caregivers upon patients' presentation: sex, age and type of referral. We also checked for differences in door-to-ECG time during the different shifts.

As there was no prespecified plan to adjust for multiple comparisons, 95\% confidence intervals were not adjusted for multiple comparisons and inferences drawn from them may not be reproducible. Descriptive analyses were performed using Graph Pad Prism Version 6.0 (Prism 6 for Mac OS X; GraphPad Software, Inc., La Jolla, CA) and multivariable median regressions were conducted using Stata version 16.1 (StataCorp, College Station, Texas).

\section{Results}

Baseline characteristics

1101 patients met the inclusion criteria. Of those, 362 (33\%) had hCAD and 739 (67\%) had no hCAD. Of those, 351 patients received CAG (172 [49\%] patients with hCAD and 179 [51\%] patients with no hCAD).

Of all included patients, the ones with hCAD were older than patients with no hCAD (74 years [IQR 65-82] vs. 60 years [IQR 46-74]; $p<0.001) .31 \%$ in the knownCAD group and $46 \%$ in the no-known-CAD group were female $(p<0.001)$.

Compared with patients with no-hCAD, patients with hCAD had higher risk-scores (Global Registry of Acute Coronary Events [GRACE] score: 125 points [IQR 104147] vs. 93 points [IQR $62-125] ; p<0.001$ and Thrombolysis In Myocardial Infarction,[TIMI] Score 2 points [IQR 2-3] vs. 1 point [IQR 0-2]; $p<0.001$ ), more cardiovascular risk factors (Diabetes mellitus $28 \%$ vs. $14 \%$; $p<0.001$, hypercholesterinaemie $51 \%$ vs. $13 \% ; p<0.001$, arterial hypertension $80 \%$ vs. $44 \%$; $p<0.001$ and family hCAD $18 \%$ vs. $13 \% ; p<0.001)$ and more co-morbidities (chronic kidney disease $26.8 \%$ vs. $4.5 \%$; $p<0.001$, history of stroke $11.4 \%$ vs. $3.7 \%$; $p<0.001$, peripheral arterial disease $12.9 \mathrm{v} \%$ vs. $2.3 \% ; p<0.001$, heart failure $12.1 \%$ vs. $1.5 \% ; p<0.001)$.

Patients with hCAD were more likely to be referred to the hospital by emergency medical services (238 patients [66\%] vs. 362 patients $[49 \%] ; p<0.0001)$ while patients with no hCAD more frequently were self-referrals (56 patients [15\%] vs. 223 patients [30]; $p<0.001)$.

\section{Analysis of time intervals Pre-admission}

There was statistical significant difference in the time from initial symptoms to ED admission between patients with hCAD and patients with no-hCAD (0.8 h [IQR 0.6$11]$ vs. $12 \mathrm{~h}$ [5-18]; $p<0.001)$. Patients with hCAD were more likely to receive a pre-clinical ECG (31.8\% [N115] vs. 20.7\% [N153]; $p<0.001$ ) (Table 1).

\section{Following admission to ED}

The total time in the ED was significantly higher for patients with known hCAD when compared with patients with no known hCAD (7.5 h [IQR 5-10] vs. 6.4 h [IQR 5-11]; $p<0.001)$. Patients with known hCAD had significant shorter door-to-ECG time after admission when compared to patients with no known hCAD (20 min [IQR 13-30] vs. $22 \mathrm{~min}$ [IQR 14-37]; $p<0.001$ ) (Fig. 1).

There was a statistically significant difference in doorto-ECG time during different shifts: Night- and earlyshift $(-4.85 \mathrm{~min}$. [ $p<0.001 ; 95 \mathrm{CI}-7.39$ to -2.32$])$. (Table 3).

Patients with known hCAD were more likely to be admitted during the early shift (48\% [N175] vs. $42 \%$ [N308]; $p=0.04$ ) (Table 2).

\section{Multivariable regression analysis}

Multivariable regression analysis of all patient data with the dependent variable being door-to-ECG time showed a relevant association of hCAD with door-to-ECG time with a coefficient of $-3 \min (p=0.007 ; 95 \% \mathrm{CI}-5.16$ to $-0.84 \mathrm{~min})$. This association prevailed when only patients admitted via health care professionals (e.g. emergency doctor) were considered with $-3.9 \min (\mathrm{n}=598$; $p=0.006 ; 95 \% \mathrm{CI}-6.7$ to $-1.1 \mathrm{~min}$ ).

Data analysis also showed that patient age correlated with delay in door-to-ECG time $(0.007 \mathrm{~min}[p=0.012$; 95\% CI 0.02-0.13]). Admission to ED via emergency services was associated with a shorter time to ECG $(-3.53 \mathrm{~min}$ [95\% CI -5.69 to $-1.38 \mathrm{~min} ; p=0.001])$ (Table 3).

If only patients admitted via emergency services were considered $(\mathrm{N}=598)$, hCAD was the only factor associated with a shorter door-to-ECG time $(-3.93 \mathrm{~min}$ [ $p=0.006 ; 95 \% \mathrm{CI}-6.74$ to -1.12$]$ ) (Table 3B).

\section{Outcome analysis}

351 patients were referred to CAG after ED admission, 179 (51\%) with hCAD and 172 (49\%) with no hCAD. Patients with hCAD referred to CAG were more likely to be male when compared to patients with no hCAD (70\% [121 N] vs. $52 \%$ [93]; $p<0.001)$. There was no statistically 
Table 1 (A) Baseline characteristics of included patients, (B) door-to-ECG time in included patients in general, in patients referred via emergency doctors, ambulance services and self-referral respectively

\begin{tabular}{|c|c|c|c|c|c|}
\hline \multirow[t]{2}{*}{$N$} & \multicolumn{2}{|c|}{ No hCAD } & \multicolumn{2}{|c|}{ HCAD } & \multirow[t]{2}{*}{$p$} \\
\hline & 739 & & 362 & & \\
\hline \multicolumn{6}{|l|}{ (A) } \\
\hline Age in years & 60 & $(46-74)$ & 74 & $(65-82)$ & $<0.001^{\mathrm{a}}$ \\
\hline Grace score & 93 & $(62-125)$ & 125 & $(104-147)$ & $<0.001^{\mathrm{a}}$ \\
\hline Creatinin in mg/dl & 0.9 & $(0.8-1.1)$ & 1.1 & $(0.9-1.4)$ & $<0.001^{\mathrm{a}}$ \\
\hline Female in \% (N) & 45.7 & (338) & 31.2 & $(113)$ & $<0.001^{b}$ \\
\hline Pre hospital ECG in \% (N) & 20.7 & (153) & 31.8 & (115) & $<0.001^{b}$ \\
\hline Diabetes mellitus in \% (N) & 13.7 & $(101)$ & 27.9 & $(101)$ & $<.0001^{\mathrm{b}}$ \\
\hline Arterial hypertension \% (N) & 44.1 & $(326)$ & 79.6 & (288) & $<0.001^{b}$ \\
\hline Chronic kidney failure \% (N) & 5.1 & (38) & 25.7 & (93) & $<0.0001^{b}$ \\
\hline Positive family history for CV events in \% (N) & 13 & 96 & 18.2 & (66) & $0.02^{\mathrm{b}}$ \\
\hline Hyperlipidaemia in \% (N) & 12.7 & 94 & 51.4 & (186) & $<0.001^{b}$ \\
\hline History of smoking in \% (N) & 23.7 & 175 & 20.2 & (73) & $0.2^{\mathrm{b}}$ \\
\hline TIMI score & 2 & $(2-3)$ & 1 & $(0-2)$ & $<0.0001^{\mathrm{a}}$ \\
\hline Peripheral artery disease in \% (N) & 2 & (16) & 14 & (49) & $<0.0001^{b}$ \\
\hline Emergency admission ${ }^{c}$ in \% (N) & 49 & (362) & 66 & (238) & $<0.001^{\mathrm{b}}$ \\
\hline Self referral in \% (N) & 30 & $(223)$ & 15 & $(56)$ & $<0.001^{b}$ \\
\hline \multicolumn{6}{|l|}{ Time point of admission } \\
\hline Morning shift in \% (N) & 42 & (308) & 48 & $(175)$ & $0.04^{b}$ \\
\hline Night shift in \% (N) & 20 & $(150)$ & 18 & (65) & $0.4^{\mathrm{b}}$ \\
\hline Late shift (in \% (N) & 38 & $(281)$ & 34 & $(122)$ & $0.2^{b}$ \\
\hline \multicolumn{6}{|l|}{ (B) } \\
\hline Time-to-EKG in minutes & 22 & $(14-37)$ & 20 & $(13-30)$ & $<0.001^{\mathrm{a}}$ \\
\hline Via emergency doctor time-to-EKG in minutes & 2 & $(0-15)$ & 16 & $(12-23)$ & $<0.001^{\mathrm{a}}$ \\
\hline Via ambulance service time-to-EKG in minutes & 20 & $(12-35)$ & 19 & $(14-25)$ & $<0.001^{\mathrm{a}}$ \\
\hline Self-referral time-to-EKG in minutes & 32 & $(20-51)$ & 30 & $(16-49)$ & $0.5^{\mathrm{a}}$ \\
\hline Total time in CPU in hours & 6.4 & $(5-11)$ & 7.5 & $(5-10)$ & $<.0001^{\mathrm{a}}$ \\
\hline First symptoms to admission in hours & 12 & $(5-18)$ & 0.8 & $(0.6-11)$ & $<0.001^{\mathrm{a}}$ \\
\hline
\end{tabular}

$\mathrm{N}=$ Number of patients; $p$ values refer to the comparison between the hCAD negative and the hCAD positive patients

a Presented as median \pm interquartile range

${ }^{b}$ Based on chi-square test/Fisher's exact test as appropriate for categorical variables

' Referred via Emergency Doctor, Rescue services, airborn rescue services/air rescue

significant difference in number of percutaneous transluminal coronary angioplasty $(42 \%[76 \mathrm{~N}]$ vs. $45 \%[79 \mathrm{~N}]$; $p=0.4)$ or percutaneous coronary intervention (42\% $(76 \mathrm{~N})$ vs. $44 \%[75 \mathrm{~N}] p=0.8)$ performed in patients with no hCAD and hCAD accordingly. Patients with hCAD were statistically significantly more often discharged with the final diagnose being NSTEMI $(45 \%$ [62 N] vs. $25 \%$ [36 N]). NSTEMI patients with no hCAD had a statistically significant higher levels of creatinkinase within $48 \mathrm{~h}$ after admission (357 U/I [162-791] vs. 245 U/I [110385]; $p=0.03)$. There was no statistically significant difference in patients with no hCAD being discharged with the diagnose "Stable Disease" or "Exclusion of relevant CAD" (53\% (91 N) vs. 56\% [101 N]; $p=0.5)$. Patients with hCAD had a statistically significant longer doorto-CAG time (33.3 h [IQR 9-68] vs. $24.5 \mathrm{~h}$ [IQR 5-54]; $p=0.01$ ) when compared to patients with no hCAD. This statistically significant difference prevailed when looking at patients with final diagnose: NSTEMI $(29.5 \mathrm{~h}$ [IQR $10-48$ ] vs. 20.7 h [IQR 6-33]; $p=0.01$ ) (Table 2).

\section{Discussion}

In this single center, retrospective, observational registry, we evaluate the influence of a known hCAD on door-toECG time in patients referred to the ED for cardiac evaluation and in a subgroup analyses of patients transferred 


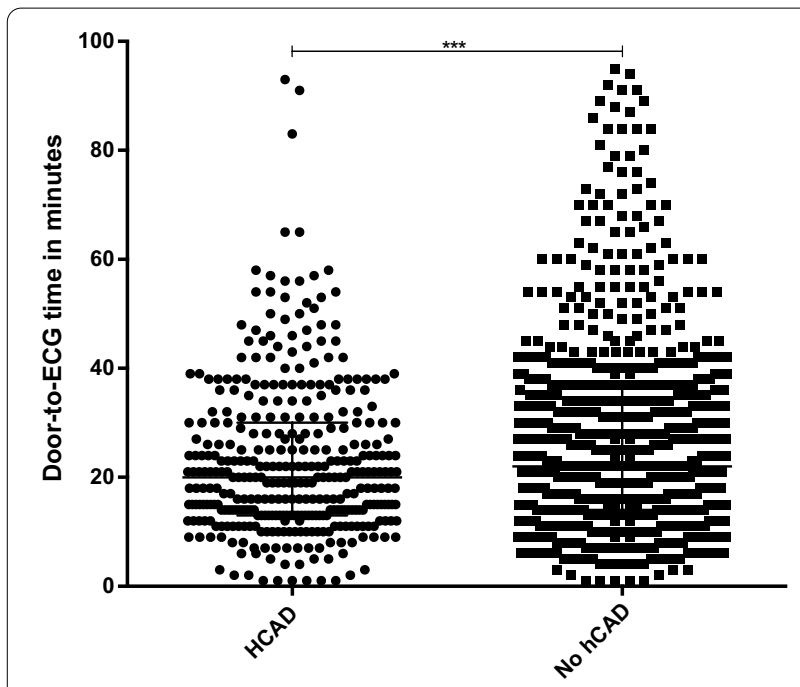

Fig. 1 Time to ECG in patients with hCAD and no hCAD respectively. Data are presented as Scatter blocks with median and interquartile range. ${ }^{* * *} p<0.001 ; C A D$ coronary artery disease to CAG, on door-to-CAG time when compared to CADnaïve patients.

We show that hCAD is associated with a decrease in door-to-ECG time especially in patients admitted via emergency services. This increased awareness for recurrent cardiovascular events in medical personnel might be due to the frequency healthcare professionals are confronted with cardiovascular disease. This presumption is validated by several studies showing that patients with a history of $\mathrm{CV}$ events are more likely to experience a recurrent $\mathrm{CV}$ event especially since they often have an elevated cardiovascular risk profile. It is vital to understand what influences door-to-ECG time after emergency admission to ED as studies show, that door-to-ECG time is one of the main controllable factors influencing doorto-balloon time $[16,17]$.

Especially since the new guidelines push for an increased awareness of high-risk NSTEMI patients who are eligible for fast-track coronary angiography, a rapid ECG after ED admission gained a central role in the decision for early reperfusion therapy and a 10 min rule for

Table 2 (A) Baseline characteristics of patients who received coronary angiography, (B) outcome of patients who received coronary angiography

\begin{tabular}{|c|c|c|c|c|c|}
\hline \multirow[t]{2}{*}{$\mathbf{N}$} & \multicolumn{2}{|c|}{ No hCAD } & \multicolumn{2}{|c|}{ HCAD } & \multirow[t]{2}{*}{$p$} \\
\hline & 179 & & 172 & & \\
\hline \multicolumn{6}{|l|}{ (A) } \\
\hline Age in years & 67 & $(57-75)$ & 73 & $(63-80)$ & $<0.001^{\mathrm{a}}$ \\
\hline Troponin $T \mu \mathrm{g} / \mathrm{L}$ at admission & 0.02 & $(0.01-0.15)$ & 0.02 & $(0.01-0.05)$ & $0.3^{\mathrm{a}}$ \\
\hline GRACE score & 108 & $(88-134)$ & 125 & $(105-150)$ & $<0.001^{\mathrm{a}}$ \\
\hline Male in \% (N) & 52 & (93) & 70 & $(121)$ & $<0.001^{b}$ \\
\hline TIMI score & 1.9 & $( \pm 1.1)$ & 2.4 & $( \pm 0.93)$ & $<0.001^{c}$ \\
\hline \multicolumn{6}{|l|}{ (B) } \\
\hline Admission to coronary angiography in hours & 24.5 & $(5-54)$ & 33.3 & $(9-68)$ & $0.01^{\mathrm{a}}$ \\
\hline \multicolumn{6}{|l|}{ Results of angiogram } \\
\hline Percutaneous transluminal coronary angioplasty in \% (N) & 42 & (76) & 45 & (79) & $0.4^{\mathrm{b}}$ \\
\hline Percutaneous coronary intervention in \% (N) & 42 & (76) & 44 & (76) & $0.8^{\mathrm{b}}$ \\
\hline Number of stents & 1 & $(1-2)$ & 1 & $(1-2)$ & $0.7^{\mathrm{a}}$ \\
\hline Number of involved vessels & 2 & $(1-3)$ & 3 & $(2-3)$ & $0.004^{\mathrm{a}}$ \\
\hline Stable disease/exclusion of relevant CAD in \% (N) & 56 & $(101)$ & 53 & (91) & $0.5^{\mathrm{b}}$ \\
\hline NSTEMI in \% (N) & 25.1 & (45) & 36 & (62) & $0.02^{b}$ \\
\hline NSTEMI admission to coronary angiography in hours & 20.7 & $(6-33)$ & 29.5 & $(10-48)$ & $0.01^{\mathrm{a}}$ \\
\hline STEMI in \% (N) & 8.4 & (15) & 7.5 & (6) & $0.8^{b}$ \\
\hline NSTEMI patients CK-max. (U/l) within $48 \mathrm{~h}$ after admission & 357 & $(162-791)$ & 245 & $(110-385)$ & $0.03^{b}$ \\
\hline
\end{tabular}

$\mathrm{N}=$ Number of patients; $p$ values refer to the comparison between the hCAD negative and the hCAD positive patients

CAD coronary artery disease, NSTEMI non-ST segment elevation myocardial infarction, STEMI ST-segment elevation myocardial infarction, CK creatinkinase

a Presented as median \pm interquartile range

${ }^{\mathrm{b}}$ Based on chi-square test/Fisher's exact test as appropriate for categorical variables

c Presented as mean \pm standard deviation 
Table 3 Multivariable median regression analysis (A: all patients. $N=1.101$; B: Emergency patients ${ }^{\mathrm{a}}$ only, $N=598$ ). Dependent variable: Door-to-ECG time

\begin{tabular}{llrrr}
\hline & Coefficient & $\boldsymbol{p}$ value & $\mathbf{9 5 \%} \mathrm{Cl}$ & \\
\hline (A) & & & & \\
HCAD & -3.00 & 0.007 & -5.16 & -0.84 \\
Emergency admission $^{\mathrm{a}}$ & -3.53 & 0.001 & -5.69 & -1.38 \\
& Early shift & & & \\
Night shift & -4.85 & $<0.001$ & -7.39 & -2.32 \\
Late shift & -1.76 & 0.159 & -4.20 & 0.69 \\
Female & 0.48 & 0.659 & -1.65 & 2.61 \\
Age & 0.07 & 0.012 & 0.02 & 0.13 \\
(B) & & & & \\
HCAD & -3.93 & 0.006 & -6.74 & -1.12 \\
Early shift & Reference & & & \\
Night shift & -2.67 & 0.154 & -6.36 & 1.01 \\
Late shift & -1.95 & 0.249 & -5.28 & 1.37 \\
Female & 0.77 & 0.608 & -2.17 & 3.70 \\
Age & 0.05 & 0.229 & --0.03 & 0.12 \\
\hline
\end{tabular}

${ }^{a}$ Referred via Emergency Doctor, Rescue services, airborn rescue services/air rescue

door-to-ECG time is recommended in guidelines [7]. Our results show a significantly reduced door-to-ECG time when compared to other studies which may be due to the implementation of a chest pain unit (CPU) into ED management in early 2013 [18]. However, still only a fraction received ECG within the suggested time frame especially in self-referral patients [19]. This calls for an increased effort for timely ECG in the ED irrespective of hCAD but may be associated with the fact that a relevant part of included patients received pre-clinical ECGs via the emergency doctor and that in self-referral patients we included the time of triage.

Also associated with door-to-ECG time was patient age with older patients experiencing a delay in door-to-ECG time by the year. Several studies have shown, that older patients are at risk to receiving an assignment of an inappropriately low triage level possibly due to different reference values of vital signs, atypical disease presentations, or the presence of cognitive impairment [20]. Nevertheless rapid diagnostic in ED should be facilitated irrespective of age.

Our data also shows, that in patients with hCAD the referral to subsequent coronary angiography is prolonged despite an increased GRACE score suggesting an early invasive strategy.

This delay in invasive diagnostic might be owed to the fact that patients with hCAD presented with a more complex array of not only cardiovascular risk factors but relevant comorbidities which makes it more difficult for the clinician to get an overview on the patient history and put his current symptoms into perspective [21]. Nonetheless, as those patients presented with a higher TIMIand GRACE score, they were likely to profit from a more rapid approach. This shows that risk score assessment might be underutilized in ED despite being a useful tool to single out high-risk patients eligible for fast-line diagnostic [12, 13].

Also, in accordance with other studies, our results show that patients with renal impairment are less likely to receive early CAG. This is most likely of mixed genesis including an uncertainty as to the interpretation of troponin measurements in patients with chronic kidney disease (CKD), atypical presentation of symptoms and concerns regarding acute kidney failure after contrast medium induced acute kidney injury [22-24]. Nevertheless, international guidelines also support an early invasive management strategy in CKD patients with suspected AMI due to a two-fivefold greater risk of death after AMI and ED personnel should be briefed accordingly [25].

As a result of the aforementioned delayed referral to CAG, it is incidental that patients with hCAD spent longer time at the ED. Reducing the length of stay through accelerating door-to-ECG time in all patients could be a powerful tool to cost saving in ED [26].

Our data show, that patients with hCAD had increased likelihood for acute myocardial infarction when compared with patients with no hCAD. This is supported by findings in other studies [27]. It may be partly explained by the fact that patients with hCAD had more cardiovascular risk factors and understandable since patients with hCAD had higher probability for recurrent ischemic events determent by TIMI- and GRACE score [13]. Nevertheless, hCAD is easily retrievable information for patient triage at ED.

We suspected and there are studies elaborating, that patients with hCAD due to the fact that they are able to identify symptoms quicker and arrive at ED faster when compared to CAD naïve patients [28]. Consequently, our data show a statistically significant difference between patients with hCAD and patients with no hCAD in respect to time from beginning of symptoms to admission to ED. Arguably, this might be due to the fact that patients with a hCAD are eligible to recall classical symptoms such as angina pectoris faster and act accordingly.

A similar picture emerges when we examined the modus of referral to the hospital in the different groups with patients with hCAD being far more likely to call and be referred by emergency medical services speaking for an increased awareness in those patients. Consequently those patients were more likely to receive a preclinical 
ECG, which might improve their outcome as some studies suggest [16].

Finally, our results show, that door-to-ECG time also depends on the shift during which patients are admitted to the ED. Especially at night with a smaller number of patients admitted door-to-ECG is significantly reduced. Nevertheless, in patients referred via emergency services, hCAD proofed to be the only factor statistically significant associated with shorter door-to-ECG time.

\section{Limitations}

Based on the presented results we plan to initiate an interventional study to improve door-to-ECG time in self-referral patients. This is a retrospective observation and we tried to depict all day routine in ED. Consequently we didn't differentiate between acute coronary syndrome and chest pain. The majority of patients with ST-myocardial infarction are not admitted via ED but directly in the cardiac catheterization laboratory therefore we could not include them. The time frame concerning begin of symptoms was taken from patients memory and is subject to individual deviations. We did include all patients admitted to ED and tried to select via full-text search, however there is still the possibility that patients were admitted for e.g. neurological or orthopaedic diagnostic and developed symptoms in the ED. All patients had hCAD but differ in presentation. That means we also included patients who did undergo elective CAG for example through initiation of a cardiologist. Therefore, such patients wouldn't know the symptoms of ACS. We did not include regression analysis for influence of hCAD on time-to-CAG since there would have been too many confounders.

\section{Conclusion}

Our observational data from a single centre registry show, that hCAD prior admission to ED is associated with a shorter door-to-ECG time. Although patients with hCAD were more often high-risk patients with a higher GRACE score, more co-morbidities and a higher cardiovascular risk profile and would therefore benefit from an early invasive strategy, they are referred to CAG later. Whether this influences patient outcome needs to be evaluated in further clinical trials. In patients referred to the ED for cardiac evaluation, timely ECG diagnostic should be facilitated irrespective of hCAD.

\section{Abbreviations}

ED: Emergency department; ECG: Electrocardiogram; CAD: Coronary artery disease; hCAD: History of coronary artery disease; CAG: Coronary angiography; AMI: Acute myocardial infarction; PAD: Peripheral artery disease; FMC: First medical contact; CV: Cardiovascular.

\section{Acknowledgements}

DD is members of SFB1425, funded by the Deutsche Forschungsgemeinschaft (DFG, German Research Foundation) - Project \#422681845. This work was supported by the DFG, German Research Foundation (Research Fellowship HE 8679/1-1:1 to L. A. Heger)

\section{Authors' contributions}

$\mathrm{LH}, \mathrm{IA}$, and HJB designed the study. $\mathrm{LH}$ and TG collected the data. TG, LH, KK, CBO analysed the data. $\mathrm{LH}, \mathrm{CBO}, \mathrm{TG}, \mathrm{HJB}$ and IA wrote the manuscript. CB, TW, $\mathrm{MH}, \mathrm{MR}, \mathrm{PMS}$ and PD reviewed and corrected the paper. All authors contributed to the article and approved the submitted version. All authors read and approved the final manuscript.

Funding

Open Access funding enabled and organized by Projekt DEAL.

\section{Availability of data and materials}

The datasets generated for this study are available on reasonable request to the corresponding author.

\section{Declarations}

\section{Ethics approval and consent to participate}

The protocol of this study conforms to the ethical guidelines of the 1975 Declaration of Helsinki and was henceforth approved by the institutional ethical committee of university of Freiburg (permit numbers EK99/17), which also waived the requirement to obtain any informed consent.

\section{Consent for publication}

Not applicable. As this is a retrospective study informed consent was not obtained from all individual participants included in the study. This procedure is backed by the institutional ethical committee of university of Freiburg (permit numbers EK99/17).

\section{Competing interests}

All authors have no conflicts of interest to declare that are relevant to the content of this article.

\section{Author details}

${ }^{1}$ Department of Cardiology and Angiology I, Heart Center Freiburg University, Faculty of Medicine, University of Freiburg, Freiburg, Germany. ${ }^{2}$ Institute of Medical Biometry and Statistics, Faculty of Medicine and Medical Center, University of Freiburg, Freiburg, Germany. ${ }^{3}$ Center of Big Data Analysis in Cardiology (CeBAC), Heart Center Freiburg University, Department of Cardiology and Angiology I, Faculty of Medicine, University of Freiburg, Freiburg, Germany. ${ }^{4}$ Departement of Emergency Medicine, University Medical Center Freiburg, Medical Faculty, University of Freiburg, Freiburg, Germany. ${ }^{5}$ Department of Cardiology and Medical Intensive Care, Augustinerinnen Hospital, Academic Teaching Hospital University of Cologne, Cologne, Germany.

Received: 24 June 2021 Accepted: 26 August 2021

Published online: 07 October 2021

\section{References}

1. Christ M, Popp S, Pohlmann H, Poravas M, Umarov D, Bach R, et al. Implementation of high sensitivity cardiac troponin T measurement in the emergency department. Am J Med. 2010;123(12):1134-42.

2. Fruergaard P, Launbjerg J, Hesse B, Jorgensen F, Petri A, Eiken P, et al. The diagnoses of patients admitted with acute chest pain but without myocardial infarction. Eur Heart J. 1996;17(7):1028-34.

3. Coster JE, Turner JK, Bradbury D, Cantrell A. Why do people choose emergency and urgent care services? A rapid review utilizing a systematic literature search and narrative synthesis. Acad Emerg Med. 2017;24(9):1137-49.

4. Hampton JR. Five men with chest pain. Practitioner. 1999;243(1596):227-31.

5. Amsterdam EA, Wenger NK, Brindis RG, Casey DE Jr, Ganiats TG, Holmes DR Jr, et al. 2014 AHA/ACC guideline for the management of patients 
with non-ST-elevation acute coronary syndromes: a report of the American college of cardiology/American heart association task force on practice guidelines. J Am Coll Cardiol. 2014;64(24):e139-228.

6. Steg PG, James SK, Gersh BJ. 2012 ESC STEMI guidelines and reperfusion therapy: evidence-based recommendations, ensuring optimal patient management. Heart. 2013;99(16):1156-7.

7. Ibanez B, James S, Agewall S, Antunes MJ, Bucciarelli-Ducci C, Bueno $H$, et al. 2017 ESC guidelines for the management of acute myocardial infarction in patients presenting with ST-segment elevation: the task force for the management of acute myocardial infarction in patients presenting with ST-segment elevation of the European Society of Cardiology (ESC). Eur Heart J. 2018;39(2):119-77.

8. Collet JP, Thiele H, Barbato E, Barthélémy O, Bauersachs J, Bhatt DL, et al. 2020 ESC Guidelines for the management of acute coronary syndromes in patients presenting without persistent ST-segment elevation. Eur Heart J. 2020;42:1289-367.

9. Bhatt DL, Eagle KA, Ohman EM, Hirsch AT, Goto S, Mahoney EM, et al. Comparative determinants of 4-year cardiovascular event rates in stable outpatients at risk of or with atherothrombosis. JAMA. 2010;304(12):1350-7.

10. Knuuti J, Wijns W, Saraste A, Capodanno D, Barbato E, Funck-Brentano C et al. 2019 ESC guidelines for the diagnosis and management of chronic coronary syndromes. Eur Heart J. 2020;41(3):407-77.

11. Laureano-Phillips J, Robinson RD, Aryal S, Blair S, Wilson D, Boyd K, et al. HEART score risk stratification of low-risk chest pain patients in the emergency department: a systematic review and meta-analysis. Ann Emerg Med. 2019;74(2):187-203.

12. D'Ascenzo F, Biondi-Zoccai G, Moretti C, Bollati M, Omede P, Sciuto F, et al. TIMI, GRACE and alternative risk scores in Acute Coronary Syndromes: a meta-analysis of 40 derivation studies on 216,552 patients and of 42 validation studies on 31,625 patients. Contemp Clin Trials. 2012;33(3):507-14.

13. Januzzi JL Jr, McCarthy CP. Evaluating chest pain in the emergency department: searching for the optimal gatekeeper. J Am Coll Cardiol. 2018;71(6):617-9.

14. Valadkhani S, Jalili M, Hesari E, Mirfazaelian H. Validation of the North American chest pain rule in prediction of very low-risk chest pain; a diagnostic accuracy study. Emerg (Tehran). 2017;5(1):e11.

15. Brown JP, Mahmud E, Dunford JV, Ben-Yehuda O. Effect of prehospital 12-lead electrocardiogram on activation of the cardiac catheterization laboratory and door-to-balloon time in ST-segment elevation acute myocardial infarction. Am J Cardiol. 2008;101(2):158-61.

16. Sekulic M, Hassunizadeh B, McGraw S, David S. Feasibility of early emergency room notification to improve door-to-balloon times for patients with acute ST segment elevation myocardial infarction. Catheter Cardiovasc Interv. 2005;66(3):316-9.
17. Goodacre S, Nicholl J, Dixon S, Cross E, Angelini K, Arnold J, et al. Randomised controlled trial and economic evaluation of a chest pain observation unit compared with routine care. BMJ. 2004;328(7434):254.

18. Coyne CJ, Testa N, Desai S, Lagrone J, Chang R, Zheng L, et al. Improving door-to-balloon time by decreasing door-to-ECG time for walk-in STEMI patients. West J Emerg Med. 2015;16(1):184-9.

19. Blomaard LC, Speksnijder C, Lucke JA, de Gelder J, Anten S, Schuit SCE, et al. Geriatric screening, triage urgency, and 30-day mortality in older emergency department patients. J Am Geriatr Soc. 2020;68(8):1755-62.

20. Khafaji HA, Suwaidi JM. Atypical presentation of acute and chronic coronary artery disease in diabetics. World J Cardiol. 2014;6(8):802-13.

21. Shaw C, Nitsch D, Steenkamp R, Junghans C, Shah S, O'Donoghue D, et al. Inpatient coronary angiography and revascularisation following non-STelevation acute coronary syndrome in patients with renal impairment: a cohort study using the Myocardial Ischaemia National Audit Project. PLoS ONE. 2014;9(6):e99925.

22. Lamb EJ, Hall EM, Fahie-Wilson M. Cardiac troponins and chronic kidney disease. Kidney Int. 2006;70(8):1525-6 (author reply 6).

23. Wickenbrock I, Perings C, Maagh P, Quack I, van Bracht M, Prull MW, et al. Contrast medium induced nephropathy in patients undergoing percutaneous coronary intervention for acute coronary syndrome: differences in STEMI and NSTEMI. Clin Res Cardiol. 2009;98(12):765-72.

24. Go AS, Chertow GM, Fan D, McCulloch CE, Hsu CY. Chronic kidney disease and the risks of death, cardiovascular events, and hospitalization. N Engl J Med. 2004;351(13):1296-305.

25. Vermeulen MJ, Guttmann A, Stukel TA, Kachra A, Sivilotti ML, Rowe BH, et al. Are reductions in emergency department length of stay associated with improvements in quality of care? A difference-in-differences analysis. BMJ Qual Saf. 2016;25(7):489-98.

26. Dezman ZD, Mattu A, Body R. Utility of the history and physical examination in the detection of acute coronary syndromes in emergency department patients. West J Emerg Med. 2017;18(4):752-60.

27. Bastos AS, Beccaria LM, Contrin LM, Cesarino CB. Time of arrival of patients with acute myocardial infarction to the emergency department. Rev Bras Cir Cardiovasc. 2012;27(3):411-8.

28. Sheifer SE, Rathore SS, Gersh BJ, Weinfurt KP, Oetgen WJ, Breall JA, et al. Time to presentation with acute myocardial infarction in the elderly: associations with race, sex, and socioeconomic characteristics. Circulation. 2000;102(14):1651-6.

\section{Publisher's Note}

Springer Nature remains neutral with regard to jurisdictional claims in published maps and institutional affiliations.
Ready to submit your research? Choose BMC and benefit from:

- fast, convenient online submission

- thorough peer review by experienced researchers in your field

- rapid publication on acceptance

- support for research data, including large and complex data types

- gold Open Access which fosters wider collaboration and increased citations

- maximum visibility for your research: over $100 \mathrm{M}$ website views per year

At BMC, research is always in progress.

Learn more biomedcentral.com/submissions 